\title{
Midinfrared Surface Waves on a High Aspect Ratio Nanotrench Platform
}

Takayama, Osamu; Shkondin, Evgeniy; Bodganov, Andrey; Aryaee Panah, M. Esmail; Golenitskii, Kirill; Dmitriev, Pavel; Repän, Taavi; Malureanu, Radu; Belov, Pavel; Jensen, Flemming

Total number of authors:

11

Published in:

A C S Photonics

Link to article, DOI:

10.1021/acsphotonics.7b00924

Publication date:

2017

Document Version

Peer reviewed version

Link back to DTU Orbit

Citation (APA):

Takayama, O., Shkondin, E., Bodganov, A., Aryaee Panah, M. E., Golenitskii, K., Dmitriev, P., Repän, T., Malureanu, R., Belov, P., Jensen, F., \& Lavrinenko, A. V. (2017). Midinfrared Surface Waves on a High Aspect Ratio Nanotrench Platform. A C S Photonics. https://doi.org/10.1021/acsphotonics.7b00924

\section{General rights}

Copyright and moral rights for the publications made accessible in the public portal are retained by the authors and/or other copyright owners and it is a condition of accessing publications that users recognise and abide by the legal requirements associated with these rights.

- Users may download and print one copy of any publication from the public portal for the purpose of private study or research.

- You may not further distribute the material or use it for any profit-making activity or commercial gain

- You may freely distribute the URL identifying the publication in the public portal 


\title{
Midinfrared Surface Waves on a High Aspect Ratio Nanotrench Platform
}

\author{
Osamu Takayama, ${ }^{\dagger}$ Evgeniy Shkondin, ${ }^{\dagger+}$ Andrey Bodganov, ${ }^{\S}$ M. Esmail Aryaee Panah, ${ }^{\dagger}$ \\ Kirill Golenitskii, $"$ Pavel Dmitriev, ${ }^{\S}$ Taavi Repän, ${ }^{\dagger}$ Radu Malureanu, ${ }^{\dagger}$ Pavel Belov, ${ }^{\S}$ Flemming Jensen, ${ }^{\ddagger}$ \\ and Andrei V. Lavrinenko* ${ }^{*} \dagger$
}
${ }^{\dagger}$ DTU Fotonik-Department of Photonics Engineering, Technical University of Denmark, Ørsteds Plads 343, DK-2800 Kgs. Lyngby, Denmark
${ }^{\ddagger}$ DTU Danchip - National Center for Micro- and Nanofabrication, Technical University of Denmark, Ørsteds Plads 347, DK-2800 Kgs. Lyngby, Denmark
${ }^{\S}$ International Research Centre for Nanophotonics and Metamaterials, ITMO University, 197101 St. Petersburg, Russia
"Ioffe Institute, Russian Academy of Sciences, 194021 St. Petersburg, Russia

\section{Supporting Information}

ABSTRACT: Optical surface waves, highly localized modes bound to the surface of media, enable manipulation of light at nanoscale, thus impacting a wide range of areas in nanoscience. By applying metamaterials, artificially designed optical materials, as contacting media at the interface, we can significantly ameliorate surface wave propagation and even generate new types of waves. Here, we demonstrate that high aspect ratio (1:20) grating structures with plasmonic lamellas in deep nanoscale trenches, whose pitch is $1 / 10-1$ / 35 of a wavelength, function as a versatile platform supporting both surface and guided bulk infrared waves. The surface waves exhibit a unique combination of properties: directionality, broadband existence (from $4 \mu \mathrm{m}$ to at least $14 \mu \mathrm{m}$ and beyond) and high localization, making them an attractive tool for effective control of light in an extended range of infrared frequencies.
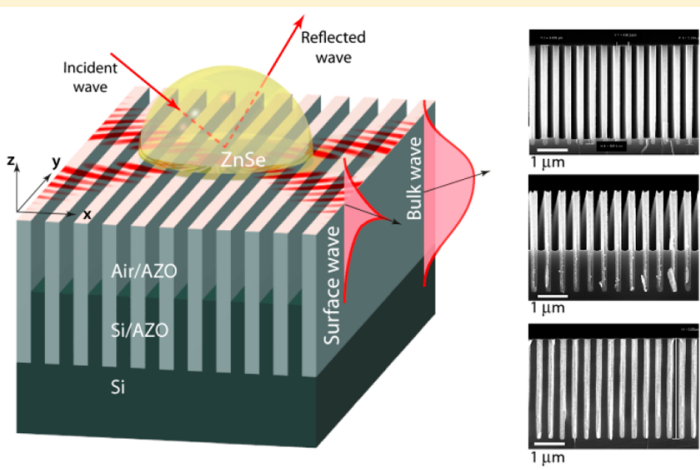

KEYWORDS: hyperbolic metamaterial, Dyakonov surface waves, plasmonics

$\mathrm{O}$ ptical surface waves (SWs) arise at the interface of two dissimilar media with different types of permittivity or permeability, such as different polarities, anisotropies, and periodic modulation of them. ${ }^{1,2}$ Research on SWs has intensified in the past decade due to their unique properties of surface sensitivity, field localization, unusual dispersion, and polarization properties at the nanoscale, stimulating the development of planar photonics, ${ }^{3}$ where light is manipulated by directional surface waves on highly anisotropic metamaterials. The most studied SWs are the surface plasmon polaritons (SPPs) supported at the interfaces between metals and dielectrics, ${ }^{4}$ which enable effective nanophotonic devices for sensing, ${ }^{5}$ nanoguiding, ${ }^{6}$ and imaging ${ }^{7}$ based on near-field techniques. However, recently SWs based on a transparent dielectric platform have also emerged as a lowloss alternative. One example is the optical Tamm states ${ }^{8}$ or Bloch surface waves, ${ }^{9-11}$ which are supported at the termination of periodic dielectric multilayers. They do not have metal's absorption losses unlike SPPs, exhibit narrow resonances tuned by design, and can be used for biochemical sensing applications. ${ }^{10}$ Another example of lossless surface waves are the Dyakonov surface wave existing at the interfaces between anisotropic and isotropic dielectrics. ${ }^{12-15}$ To date, various types of combined SWs have been investigated such as Dyakonov-Tamm states, ${ }^{16}$ Tamm plasmons, ${ }^{17,18}$ which are essentially the mixture of two different types of SWs. This is enabled by metamaterials, artificially engineered materials and structures, ${ }^{19-21}$ because, to combine different SWs, unprecedented and extreme optical parameters are often required. In this manner, we can obtain new features by combining traits from various types of surface waves. Here, we focus on the other newly emerging combined SWs on metamaterial structures, namely Dyakonov plasmons (DPs), ${ }^{22,23}$ a combination of surface plasmons and Dyakonov waves supported at the boundaries between dielectric and hyperbolic metamaterials (HMMs). ${ }^{24}$ The diagonal components of the HMMs' permittivity tensors are of different signs, giving rise to hyperbolic isofrequency contours in the $k$ (wavevector) space accompanied by singularities in the density of optical states in an ideal lossless case. Natural material equivalents of HMMs are often referred to as indefinite media. $^{25,26}$ Characteristically, HMMs and their two-dimen-

Received: August 16, 2017

Published: October 9, 2017 

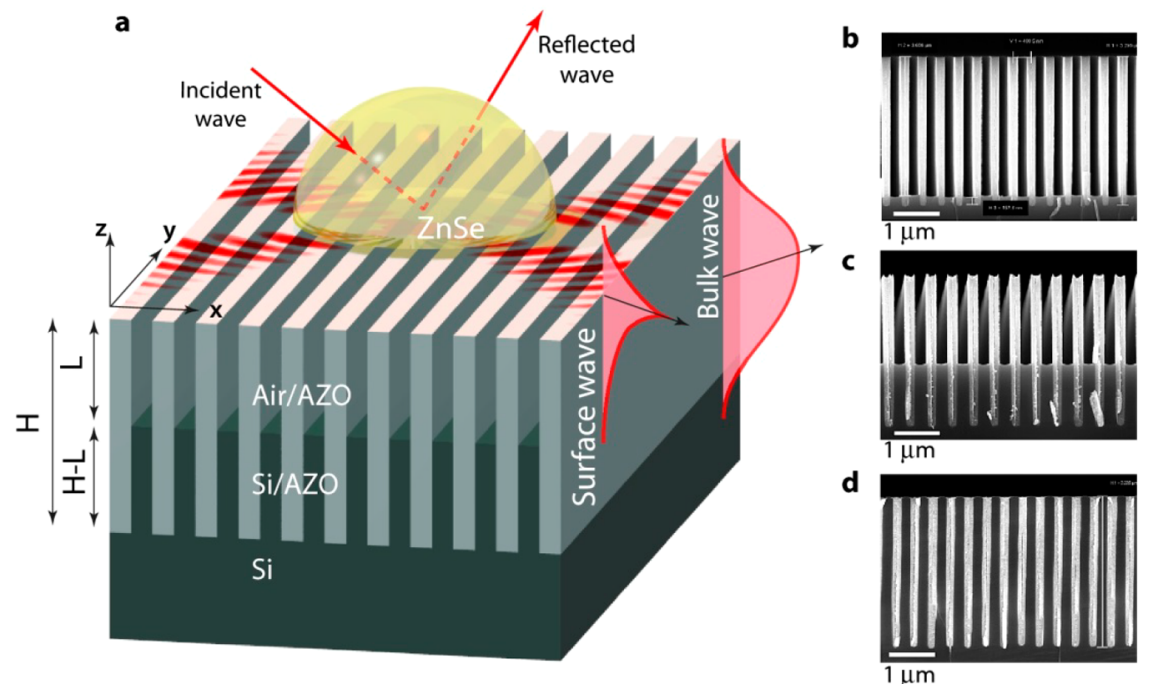

Figure 1. Hybrid bislab plasmonic trench structure. (a) Illustration of an AZO trench structure in the Otto configuration based on a ZnSe prism for the Fourier transform infrared (FTIR) spectrometer characterization. Note that there is an air gap between the ZnSe prism and the AZO trench structure. The structure drawn is a bilayer of AZO/air trenches standing on AZO/Si ones on top of the Si substrate. Dyakonov plasmons and bulk plasmon polaritons can be supported by such vertical trench structures in the hyperbolic dispersion range. The optical axis of the homogenized metamaterial is perpendicular to the trenches. Scanning electron microscope (SEM) images of trench structures: (b) AZO/air, (c) AZO/air trench on top of $\mathrm{AZO} / \mathrm{Si}$ trench, and (d) AZO/Si. The structures have pitch $\Lambda=400 \mathrm{~nm}$, the height of the entire trench structure $H=2.8-3.3 \mu \mathrm{m}$, the height of AZO/air trench $L$, filling ratio of AZO layer, $t_{\mathrm{AZO}} / \Lambda=0.5$ where $t_{\mathrm{AZO}}$ is the thickness of the AZO layer. The trench structures are standing on the $\mathrm{Si}$ substrate. Note that the height of the $\mathrm{AZO} / \mathrm{Si}$ trench is $H-L$. The pitch of trenches is deeply subwavelength $(\lambda / 10-\lambda / 35)$ allowing introduction of effective parameters in the mid-IR range. The scale bars are $1 \mu \mathrm{m}$ for all figures. In this configuration, effective ordinary permittivity $\varepsilon_{o}$ and extraordinary permittivity $\varepsilon_{e}$ are oriented along $y$ - and $z$-axes and $x$-axis, respectively.

sional analogues of metasurfaces possess a unique combination of properties including large wavevectors available due to the flat isofrequency contours, optical density of states, and anisotropy. These features lead to a wide variety of HMMs' potential applications, such as broadband enhancement in the spontaneous emission for a single photon source, ${ }^{27,28}$ subwavelength imaging, ${ }^{29}$ sensing, ${ }^{30,31}$ thermal engineering, ${ }^{27,28,32}$ and steering of optical signals. ${ }^{33,34}$ To date, various types of optical HMMs have been fabricated and characterized, including LC circuit implementation for microwave frequency, ${ }^{33}$ metal-dielectric multilayers, ${ }^{31,35-37}$ shallow metallic gratings or metasurfaces, ${ }^{34,38,39}$ metallic pillar structures ${ }^{30,40,41}$ and fishnet structures. ${ }^{32}$ Most likely, HMMs will be employed as the robust and versatile multifunctional photonic platform in the broad range of operating wavelengths from visible ${ }^{34}$ to $\mathrm{THz}$ regions $^{42}$ and even in the microwave region. ${ }^{33}$

Here, we realize a hybrid bislab metamaterial platform for midinfrared (mid-IR) surface photonics, providing flexible engineering and adiabatic tuning of directivity and dispersion of highly localized SWs, in particular DPs. Currently, infrared (IR) light with wavelengths between 2.5 and $25 \mu \mathrm{m}$ (4,000$\left.400 \mathrm{~cm}^{-1}\right)$ is used for thermal imaging and molecular detection (vibrational modes spectroscopy). ${ }^{43,44}$ In this wavelength range, DPs with large wavevectors can enhance the sensitivity of the sensing devices due to their ability to localize light at the nanoscale. As noble metals are not suitable for confining light at interfaces on mid-IR frequencies, the quest for alternative materials has been ongoing, and a large variety of materials have been proposed, such as transparent conductive oxides, ${ }^{43,45}$ doped III-V semiconductors, ${ }^{43} 2 \mathrm{D}$ materials [graphene, hexagonal boron nitride (hBN), etc.], ${ }^{46-48}$ and polar materials such as large area patterned $\mathrm{SiC}^{49-5}$

Directivity of SWs can be controlled by changing wavelengths and the dielectric environment on the interface of the metamaterial platform, making it unique for the manipulation of light on the surface. No conventional approaches for the fabrication of HMM, including multilayer stacks ${ }^{31,35-37}$ and wire medium, ${ }^{30,40,41}$ are able to provide a large area of the working interface of the platform that supports DPs. Some of the $2 \mathrm{D}$ materials, such as $\mathrm{Bi}_{2} \mathrm{Se}_{3}{ }^{52}$ and $\mathrm{hBN},{ }^{53}$ are reported to support a phonon polariton equivalent of DPs at the limited space of their wedge for their phonon resonances in mid-IR wavelengths. To attain such anisotropy, we base the platform on a high aspect ratio (up to 1:20 and even more) trench structures (Figure 1) fabricated using a combination of atomic layer deposition (ALD) and dry etch techniques (see Figure S1 and related, Supporting Information). As a plasmonic material required for the manifestation of effective hyperbolic dispersion, we used aluminum-doped $\mathrm{ZnO}(\mathrm{AZO})$ exhibiting the plasmonic response in the near and mid-IR wavelength region. $^{45,54,55}$ AZO can be deposited by the ALD technique, $^{56,57}$ allowing a conformal and uniform coating of deep trenches. To the best of knowledge, only this fabrication method can realize high aspect ratio plasmonic trench structures, whereas silver metasurface structures with low aspect ratio have been considered and realized. ${ }^{34}$ It can also benefit from the tunability of plasmonic properties by means of adjusting the doping concentration during the deposition to optimize the loss for the wavelength region of interest.

The period of trenches $(\Lambda=0.4 \mu \mathrm{m})$ is deeply subwavelength for the mid-IR wavelength range $(\Lambda / \lambda=1$ / $10-1 / 35$ for $\lambda=4-14 \mu \mathrm{m}$ ), allowing introduction of effective parameters. By controlling the etching time and rate accurately, we can reach the desired Si etching depth. Thus, three principal designs of HMMs can be obtained (Figure $1 \mathrm{~b}-\mathrm{d}$ ): AZO trenches embedded in $\mathrm{Si}(\mathrm{AZO} / \mathrm{Si})$ or air $(\mathrm{AZO} / \mathrm{air})$ and a hybrid bislab metamaterial of $\mathrm{AZO} /$ air on top of $\mathrm{AZO} / \mathrm{Si}$. Consequently, the effective properties of the hybrid composite 

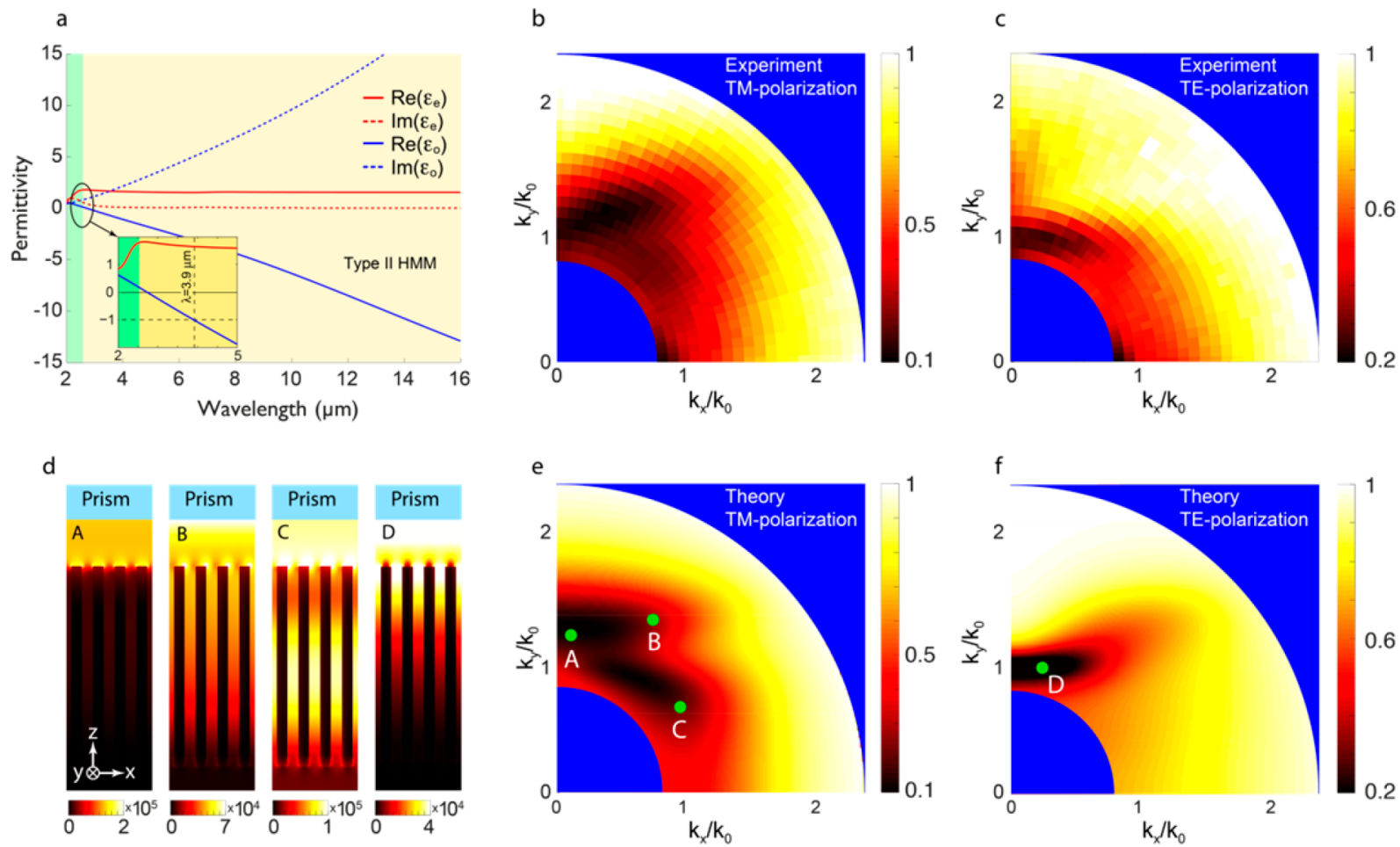

Figure 2. Observation of surface and guided bulk waves in the AZO/air trench structure. (a) Fitted real parts of effective ordinary $\varepsilon_{o}$ (blue) and extraordinary $\varepsilon_{e}$ permittivities (red) of an AZO/air trench metamaterial. The HMM range is marked in beige color. The inset shows in detail the retrieved real permittivities in the wavelength range $2-5 \mu \mathrm{m}$. The vertical dashed line in the inset designates the boundary wavelength for the DPs existence. $(\mathrm{b}, \mathrm{c})$ Experimental and $(\mathrm{e}, \mathrm{f})$ simulated reflectance in the wavevector space for $\lambda=6.0 \mu \mathrm{m}$ with TM- and TE-polarized incident light. (d) Corresponding numerical field profiles (absolute value of electric field) in points A-C from panel (e) and D from (f). The model includes the high index prism, air gap $\left(H_{\text {air }}=0.5 \mu \mathrm{m}\right)$, trench structure $(L=3.2 \mu \mathrm{m} \mathrm{AZO} /$ air on top of $H-L=0.1 \mu \mathrm{m} \mathrm{AZO} / \mathrm{Si})$, and $\mathrm{Si}$ substrate. Note that the light line of the $\mathrm{ZnSe}$ prism is located in the external blue regions in $(\mathrm{b}, \mathrm{c}, \mathrm{e}, \mathrm{f})$. Wavenumber in vacuum is $k_{0}=2 \pi / \lambda$, where $\lambda$ is the wavelength in vacuum.

structure can be accurately tuned to exhibit hyperbolic, elliptic, or epsilon-near-zero (ENZ) dispersion regimes with a plethora of ground-breaking applications including nonlinear optics. ${ }^{58,59}$ To the best of our knowledge, this is the first experimental demonstration of directional surface (Dyakonov plasmons) and guided (bulk plasmons polariton) waves in the mid-infrared range on a metamaterial platform with a high degree of flexible design parameters.

\section{SURFACE AND BULK WAVES ON AIR-PLASMONIC NANOTRENCH HYPERBOLIC METAMATERIALS}

We start with the AZO/air nanotrench structures because they are the simplest structure that supports both surface and bulk waves. To find the wavelength range, where the existence condition of DPs is met, we retrieve the effective permittivities (ordinary $\varepsilon_{o}$ and extraordinary $\varepsilon_{e}$ ) of the $\mathrm{AZO} /$ air structure from the mid-IR reflection spectra (see Figure S2-4). As shown in Figure 2a, the AZO/air trench structure has the zero crossing wavelength for $\varepsilon_{o}$ around $\lambda_{\mathrm{ENZ}}=2.5 \mu \mathrm{m}$ and becomes Type II HMM $\left(\varepsilon_{o}<0 \text { and } \varepsilon_{e}>0\right)^{27}$ within an extremely wide band at longer wavelengths. The existence condition for the DPs is $0<\varepsilon_{c}<\left|\varepsilon_{0}\right|,{ }^{23}$ where $\varepsilon_{c}$ is the permittivity of the isotropic media bordering with the HMM (in our case, $\varepsilon_{c}=1$, air). As highlighted in the inset of Figure $2 \mathrm{a}$, the condition is satisfied for $\lambda>4.0 \mu \mathrm{m}$.

One of the key assets of DPs is that they are hybrid polarization waves. For that reason, therefore, they can be excited by incident light of any polarization as opposed to conventional SPPs, which are TM waves or Bloch surface wave modes that are either transverse electric (TE) or transverse magnetic (TM) depending on the specific design. ${ }^{11}$ We study high in-plane directionality of surface waves in the AZO/air trench structures in the mid-IR wavelength interval $\lambda=2.0-$ 14.0 $\mu \mathrm{m}$ (Figure 1a and Figure 2), upon mapping the reflectance in the $k_{x}-k_{y}$ space. The incident light reaches the structure through a hemispherical $\mathrm{ZnSe}$ prism (Figure 1a) arranged in the Otto configuration. Reflection spectra are acquired under different angles of incidence and azimuthal angles with respect to the structure orientation (see such maps for $\lambda=6.0 \mu \mathrm{m}$ in Figure $2 \mathrm{~b}$ and $\mathrm{c}$ ). The zones with low reflectance outside the air light cone $k_{x}^{2}+k_{y}^{2}=k_{0}^{2}$, where $k_{0}=\omega /$ $c$, are potential candidates for SW bands because the SWs occur beyond the light line. To elucidate the nature of the different modes, we simulate the reflectance spectra of the AZO/air structure in the Otto configuration (Figure 2e and f) and map the field profiles as shown in Figure $2 \mathrm{~d}$. The reflectance spectra maps (Figure 2b, c, e, and $\mathrm{f}$ ) obtained in the hyperbolic regime at $\lambda=6.0 \mu \mathrm{m}$ clearly expose two bands. The profiles of the electric field amplitudes plotted in three characteristic points (A-C in Figure 2e) distinguish the surface and bulk waves bands (Figure $2 \mathrm{~d}$ ). At point A, the fields are strongly confined at the interface between air and the HMM featuring a SW (Figure 2d, panel A). The confinement of the SW is changed at higher in-plane wavenumbers (Figure $2 \mathrm{~d}$, panel B), suggesting the unique feature to control the localization level of the fields in the air via SWs without changing the wavelength. Plotting the field map in point $\mathrm{C}$ (Figure $2 \mathrm{~d}$, panel $\mathrm{C}$ ) enables us to identify a bulk mode in the nanotrench layer with the energy density concentrated predominantly inside the structure. The 

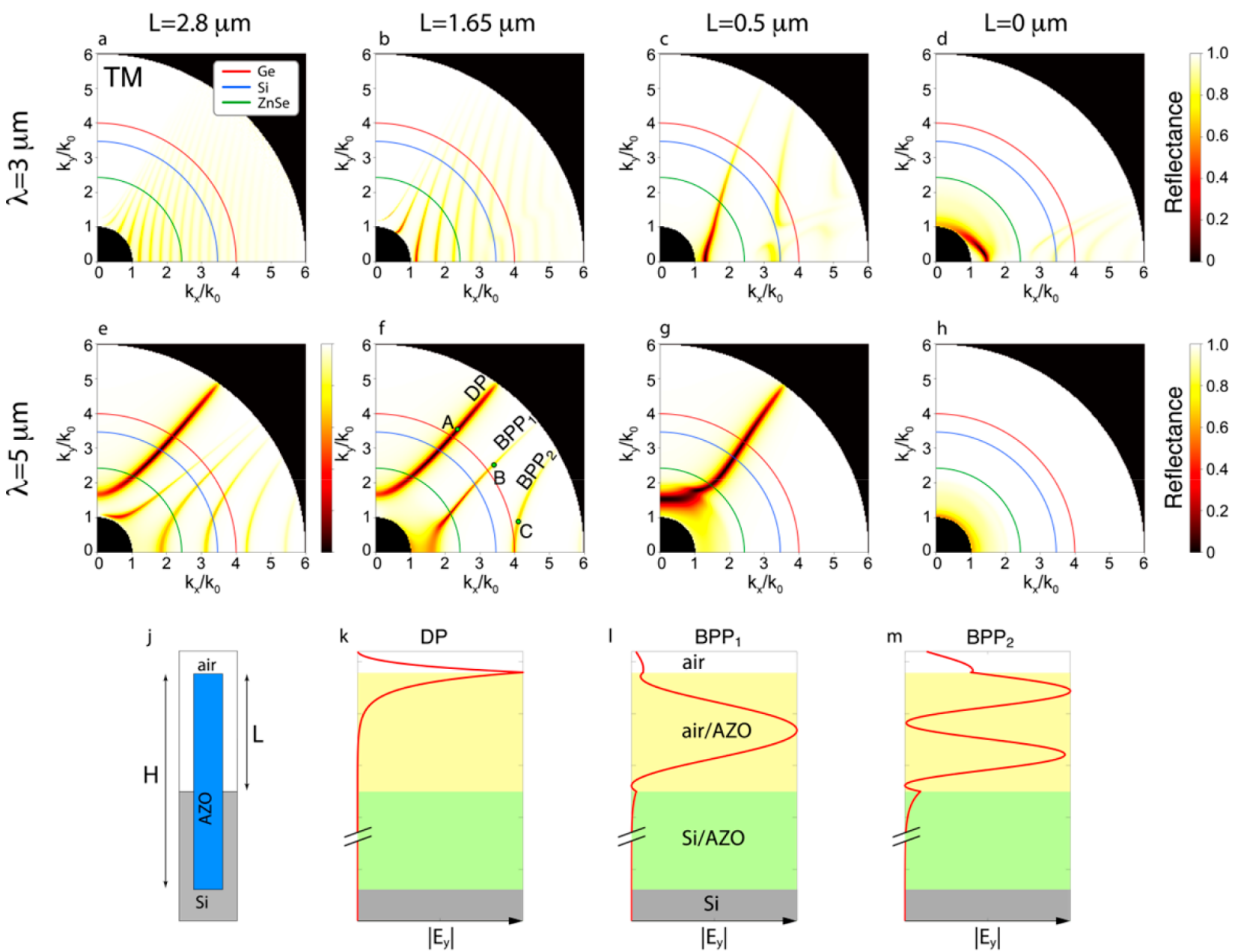

$\left|E_{y}\right|$

$\left|E_{y}\right|$

Figure 3. Transformation of band dispersion in the bislab structure for different etch depths of $\mathrm{Si}, L$. Band dispersion in numerical reflectance maps at $\lambda=3.0 \mu \mathrm{m}$ for $L=$ (a) 2.8 , (b) 1.65 , (c) 0.5 , and (d) $L=0 \mu \mathrm{m}$; at $\lambda=5.0 \mu \mathrm{m}$ for $L=$ (e) 2.8 , (f) 1.65 , (g) 0.5 , and (h) $0 \mu \mathrm{m}$, respectively. (j) Definition of $L$ and $H(=2.8 \mu \mathrm{m})$. Corresponding numerical field profiles (absolute value of electric field) in points A-C from panel (f). For the purpose of clarity in bands presentation, losses in the AZO layers are reduced by 100-times. Colored quarter-circles designate the light cones for ZnSe (green), Si (blue), and Ge (red).

surface waves are definitely classified as DPs, and volume waves correspond to guided bulk plasmon polaritons (BPPs), ${ }^{35}$ which together represent the integral feature of the HMMs. ${ }^{24}$ Although the former band appears under both TM- and TEpolarized incident light as expected for hybrid-polarized DPs (see Figure $2 \mathrm{~d}$ for field profile at point $\mathrm{D}$ in Figure $2 \mathrm{f}$ for $\mathrm{TE}$ polarization), the latter appears only for TM light as expected for SPPs and guided plasmonic modes.

The thickness of the air gap between the prism and trench structure is evaluated by fitting the simulated reflection dips with the experimental ones, giving the best matching for the airgap of $0.5 \mu \mathrm{m}$ (see details in Supporting Information, section 3 and Figure S5). Checking the wavelength dependency, we observe that DPs emerge clearly after $\lambda=4.0 \mu \mathrm{m}$, exactly as predicted by the effective parameters analysis in Figure $2 \mathrm{a}$ (the condition is satisfied at $\lambda>4.0 \mu \mathrm{m})$ and extend to the high $k$ region for longer wavelengths up to $\lambda=14.0 \mu \mathrm{m}$. Thus, their broadband existence within the Type II hyperbolic region (see Supporting Information, Figure S8 and Figure S9) is confirmed. Importantly, the qualitatively good agreement between simulated and experimental data (especially for TM polarization) suggests a remarkable robustness of DPs and BPPs against inevitable fabrication and characterization imperfections, such as the mixture of polarization.

\section{SURFACE AND BULK WAVES ON HYBRID BISLAB HYPERBOLIC METAMATERIALS}

We now discuss the tunability of a nanotrench structure by changing the vertical filling ratio of the air gaps with a residual $\mathrm{Si}$ layer component. Indeed, the AZO/air trench structure (Figure 1b) characterized above is the final product of the complete etch of interstitial $\mathrm{Si}$ between the AZO layers. In principle, the depth of $\mathrm{Si}$ etching $L$ (or the AZO/air trench height) is a free parameter, which defines the flexibility of the initial $\mathrm{AZO} / \mathrm{Si}$ template (Figure 1d) toward adjusting it to a particular wavelength range. In Figure 1c, we show one intermediate example of the controllable $\mathrm{Si}$ etch with $L=1.65$ $\mu \mathrm{m}$. Such a composite system can be interpreted as consisting of two anisotropic trench structures of thicknesses $L$ (AZO/air) and $H-L(\mathrm{AZO} / \mathrm{Si})$ placed one above another. We will refer to this configuration as the bislab model. Two extreme cases of the composition are the pure $\mathrm{AZO} / \mathrm{Si}$ slab $(L=0)$ together with the pure AZO/air slab $(L=H)$ discussed before. In general, the bislab structure can be homogenized only with respect to each of the slabs individually. Thus, it is modeled as a composition of two serial homogeneous anisotropic slabs of thicknesses $L$ and $H-L$. In such a case, simulations of the bislab model can be conducted again with a conventional transfer matrix method for 
a

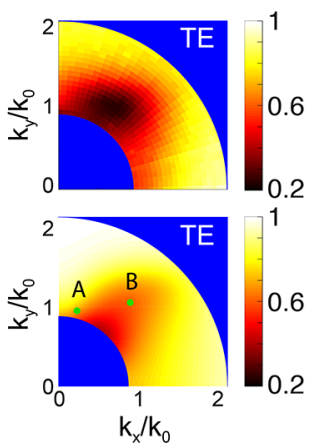

c

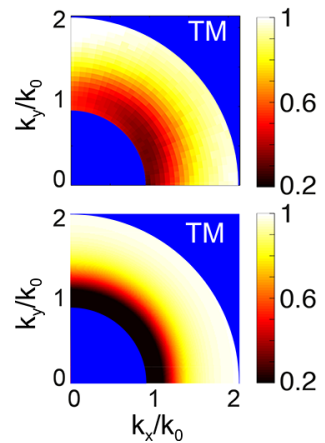

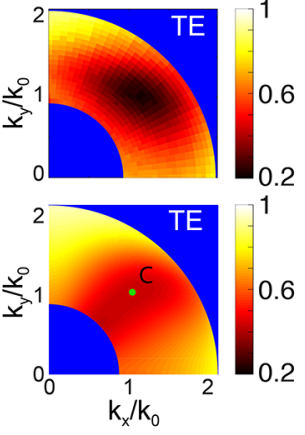

b

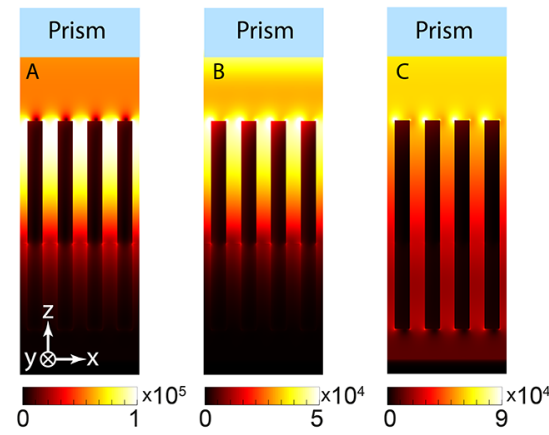

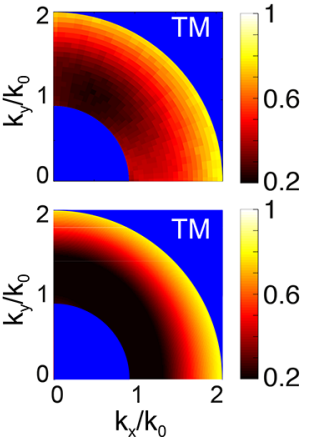

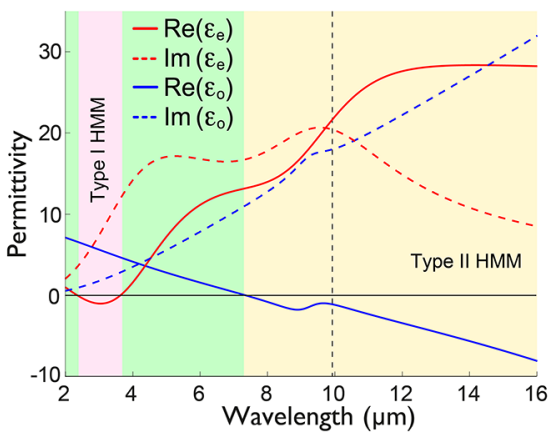

Figure 4. Surface waves on hybrid bislab trench structures. (a) Experimental and simulated reflectance in the wavevector space of the hybrid structure composed from the AZO/air $(L=1.65 \mu \mathrm{m})$ layer on the $\mathrm{AZO} / \mathrm{Si}$ layer $(H-L=1.15 \mu \mathrm{m})$ for TE-polarized incidence light at $\lambda=5.0 \mu \mathrm{m}$ (left row) and $\lambda=14.0 \mu \mathrm{m}$ (right row). (b) Corresponding electric field profiles (absolute value of electric field) at points A-C. (c) Experimental and simulated reflectance in the wavevector space of pure AZO/Si trenches $(L=0 \mu \mathrm{m}, H=3.2 \mu \mathrm{m})$ for TM-polarized light at $\lambda=5.0 \mu \mathrm{m}$ (left row) and $\lambda=14.0 \mu \mathrm{m}$ (right row). (d) Fitted real and imaginary parts of the effective ordinary $\varepsilon_{o}$ (blue) and extraordinary $\varepsilon_{e}$ permittivities (red) for the $\mathrm{AZO} / \mathrm{Si}(L=0 \mu \mathrm{m})$ trench structure. The vertical dashed line marks the boundary for the DP range.

anisotropic structures (see Supporting Information, Figure S6 and Figure S7 for accuracy analysis).

We study the gradual changes in the properties of the bislab structure upon tuning the etching depth of interstitial $\mathrm{Si}$ between the AZO layers. We first show the evolution of the guided BPP modes supported at $\lambda=3.0 \mu \mathrm{m}$ with the gradual filling of the trenches by silicon as shown in Figure $3 a-d$. We also mark the light cone for $\mathrm{ZnSe}$ (green line), Si (blue), and $\mathrm{Ge}$ (red) prisms, designating the areas within which modes can be excited in our experimental setup. For better visualization of the bands in Figure 3, the imaginary parts of effective permittivities were reduced by 100 -times, and data are presented for the TM polarization because it can excite both DPs and guided BPP modes. According to estimations, the structure supports propagation of directional high- $k$ DPs at $\lambda>$ $4.0 \mu \mathrm{m}$, so we do not observe the signature of surface waves for $\lambda=3.0 \mu \mathrm{m}$ in the deep etch case with $L=H$ (Figure 3a) corresponding to the AZO/air structure (Figure 1b). Partial filling of the interstitial voids with $\mathrm{Si}$ arranges better conditions for guided bulk waves, as shown in Figure $3 \mathrm{~b}$ (compare with experimental result in Supporting Information, Figure S12b). Further filling with $\mathrm{Si}$ up to $L=0.5 \mu \mathrm{m}$ makes the isofrequency contour remarkably flat (Figure 3c), leading to divergence-free in-plane propagation. ${ }^{60}$ With $L=0 \mu \mathrm{m}$ (Figure $3 \mathrm{~d}$ ), the case of a pure $\mathrm{AZO} / \mathrm{Si}$ slab (Figure 1d) is reached. The hyperbolic modes become lossy due to the leakage into the substrate and transit to the higher wavevectors range. Instead, the band with an elliptic isofrequency contour manifests itself close to the light cone.
We now show the evolution of the DP modes, which are supported at hybrid bislab structures for $\lambda=5.0 \mu \mathrm{m}$ (Figure $3 \mathrm{e}-\mathrm{h}$ ), where conditions for the DPs are satisfied for $\lambda>4.0$ $\mu \mathrm{m}$ as discussed previously. The DP bands are clearly visible in Figure $3 \mathrm{e}-\mathrm{g}$ as the most intensive hyperbola-like curves (see the corresponding experimental data in Supporting Information, Figure S8d and Figure S12d). Because of the exponential decay of the fields inside the structure for DPs, waves on the upper slab do not feel the presence of the lower one for a rather extended range of sizes $L$. It is remarkably exhibited in Figure $3 \mathrm{f}$ with $L=1.65 \mu \mathrm{m}$, where the influence of the $\mathrm{AZO} / \mathrm{Si}$ slab on behavior of DPs is not significant, although we see the drastically changed BPPs dispersion (see also Supporting Information, Figure S7). When the exponential tail of the DP fields reaches the lower high-index slab, it affects dispersion of the SWs, distorting the band in Figure $3 \mathrm{~g}$ for $L=0.5 \mu \mathrm{m}$. Further filling with $\mathrm{Si}$ completely ruins conditions for the DPs existence (Figure $3 \mathrm{~h}$ ).

To further confirm the bilayer system as a flexible platform for directional high- $k$ waves, we experimentally and theoretically investigate the hybrid bislab $(L=1.65 \mu \mathrm{m})$ and single $\mathrm{AZO} / \mathrm{Si}(L=0)$ samples (Figure $1 \mathrm{c}$ and $\mathrm{d})$. The hybrid system satisfies the existence condition for SWs in shorter wavelengths. For $\lambda=5.0 \mu \mathrm{m}$ (see Figure 4a, left row), the fields of SWs are located mainly inside the AZO/air trench structure (Figure $4 \mathrm{~b}$, panels $\mathrm{A}$ and $\mathrm{B}$ ), thus exhibiting a hyperbolic dispersion. However, at $\lambda=14.0 \mu \mathrm{m}$ (Figure $4 \mathrm{a}$, right row), confinement of the fields worsen (Figure $4 \mathrm{~b}$, panel $\mathrm{C}$ ), resulting in modification of the dispersion and a more complex field profile. This 
suggests that the DPs' propagation direction can be controlled efficiently over a large angular in-plane domain by varying the wavelength. The mechanism of the surface wave's dispersion modification is that for longer wavelengths the tail of the evanescent field of the SW residing on the AZO/air slab protrudes through the slab and starts to interact with the AZO/ $\mathrm{Si}$ slab, thereby leading to elliptic dispersion. Hence, to support DPs, we need a sufficiently deep trench structure, e.g., $L=1.65$ $\mu \mathrm{m}$ for $\lambda=5.0 \mu \mathrm{m}$. In this regard, the hybrid bislab model allows us to tailor the spectrally dependent directionality more efficiently than single AZO/air or AZO/Si structures separately. Such a system can exhibit Type I or Type II hyperbolic dispersion, and transition points for the hyperbolic behavior together with the relevant ENZ regime can be configured for wavelengths in the range from 2.5 (Figure $2 \mathrm{a}$ ) to $7.5 \mu \mathrm{m}$ (Figure $4 \mathrm{~d}$ ). The accuracy of the design-tuning properties is granted by the mature technology of $\mathrm{Si}$ etching and consequently precise control of the air-silicon filling fraction in the interstitial spaces between AZO lamellas. In other words, the effective properties can be accurately tuned by the height of the $\mathrm{AZO} /$ air trench structure positioned directly on top of the $\mathrm{AZO} / \mathrm{Si}$ multilayer. Such tunability is the main benefit of our hybrid bislab nanotrench structures.

In the case of the original pure $\mathrm{AZO} / \mathrm{Si}$ trenches not undergoing any $\mathrm{Si}$ etching $(L=0)$, the elliptic dispersion of TM modes (Figure 4c) is completely different from what we observed in the case of the AZO/air multilayer (see Figure $2 \mathrm{~b}$ and e). SWs exist for $\lambda>10.0 \mu \mathrm{m}$, where the structure is Type II HMM (Figure 4d). The normalized wavevector or effective mode index of the SWs on the AZO/Si structure must exceed $\left(\operatorname{Re}\left(\varepsilon_{e}\right)\right)^{1 / 2} \sim 5$, which is higher than what can be reached with assistance of a $\mathrm{ZnSe}(n=2.4)$ or even a Ge $(n=4.0)^{13}$ prism. Therefore, the bands visible in the reflection spectra mapping (Figure 4a and c) are leaky bulk modes existing in anisotropic dielectric $(\lambda=5.0 \mu \mathrm{m})$ or hyperbolic $(\lambda=14.0 \mu \mathrm{m})$ metamaterials. Again, we would like to emphasize the good qualitative correspondence of the experimental results in Figure 4 with numerical pictures from Figure $3 \mathrm{e}$ to $3 \mathrm{~h}$ despite the heavily reduced losses in modeling.

For the mid-IR wavelengths, some of two-dimensional materials are known to support surface waves and bulk modes, such as plasmons on graphene, hyperbolic phononpolaritons in hBN, and chiral plasmons on $\mathrm{MoS}_{2} \cdot{ }^{47,48}$ Recently, hyperbolic surface phonon-polaritons, which are the phononpolariton equivalent of Dyakonov plasmons, have been observed at the wedge of a topological insulator $\mathrm{Bi}_{2} \mathrm{Se}_{3}$ proven by electron energy loss spectroscopy ${ }^{52}$ and hBN by scatteringtype scanning near-field optical microscope. ${ }^{53}$ However, because of the resonance character of phonon-polaritons, the existence of most of these modes is restricted to narrow bands attributed to specific materials. On the contrary, the DPs on the trench structures are shown to exhibit broadband existence (from $4 \mu \mathrm{m}$ to at least $14 \mu \mathrm{m}$ and beyond) as well as unique properties such as directionality and controllable localization. Furthermore, our deep subwavelength nanotrenches offer the possibility to tune the surface and bulk modes in the hybrid bislab HMM platform upon tuning (1) the material properties by adjusting the amount of doping $\mathrm{Al}$ concentration in $\mathrm{ZnO}$ and (2) the effective (metamaterials) properties by controlling the thickness of $\mathrm{AZO} /$ air slab on $\mathrm{AZO} / \mathrm{Si}$ structures in the hybrid bislab configuration. Such flexibility and broad parametric space in the optimization regime enable tuning (1) of the operational wavelength from mid-IR to $\mathrm{THz}$ ranges and (2) the direction of Dyakonov plasmon propagation. In principle, further strong and fine-tuning of a given trench structure may also be activated by electric gating of the AZO layers. ${ }^{61-63}$ The demonstrated HMMs can be harnessed with $2 \mathrm{D}$ materials to hybridize optical modes from both parts because the broadband existence of DPs extends over all operating wavelengths of 2D materials in the mid-infrared region. ${ }^{47,48}$ Moreover, highly localized SWs may enhance nonlinearity of AZO layers.

In conclusion, we characterize directional surface waves supported by the deep trench structures in the mid-infrared wavelength range. The structures are based on multiple highaspect ratio (1:20) subwavelength AZO trenches embedded into the supporting $\mathrm{Si}$ substrate. Well-established $\mathrm{Si}$ etching technology is used to fabricate either a single slab with pure $\mathrm{AZO} /$ air trenches or a hybrid bislab with AZO/air trenches on top of $\mathrm{AZO} / \mathrm{Si}$ trenches. All fabrication steps are supported by the large-scale complementary metal oxide semiconductor (CMOS)-compatible highly reproducible technological approaches. An AZO/air trench structure performs as a broadband HMM. We observed two bands of directional waves classified further as surface DPs and guided BPPs according to the field confinement mapping. Existence conditions for different regimes of such directional waves can undergo very fine adjustment by tailoring the relative thicknesses of the hybrid bislab structures. The broadband hyperbolic behavior of the trench structure allows for the wavelength sweeping regime, and electrical gating of the AZO material can be an additional mechanism of the performance tuning. Our demonstration of SW behavior in the deep trench structured platform can be conceptually extended to other wavelength regimes such as the near-infrared, $\mathrm{THz}$, and even the visible one by the choice of the relevant material platform. This could enable, for instance, the use of DPs with controllable dispersion for photonics applications as routing and switching of optical signals. Indeed, optical nonlinearities are expected to be high as well in the AZO-based metamaterial. Confinement of light close to the interface can also be effectively used for midIR spectroscopy to detect traces of analyte molecules via surface wave-enhanced sensing. ${ }^{44,64}$ Furthermore, the open air volume inside the hybrid nanotrenches would be free for infiltration of analytes in gaseous and a solution environment, allowing for both surface DP and guided BPP resonance shifts. ${ }^{65}$ In such a perspective, functionalization of the AZO trench walls can be proposed as a further step for label-free biosensing experiments. Finally, fluorescence emitters could channel their radiation directly in the high directional surface modes or allow for strong-coupling of the nanotrench photonic modes with embedded exciton modes.

\section{SAMPLE FABRICATION}

$\mathrm{Al}$-doped $\mathrm{ZnO}(\mathrm{AZO})$ high aspect ratio trench structures were prepared by combining atomic layer deposition (ALD) and dry etch techniques. The use of ALD in combination with a sacrificial Si template is a novel way to create high aspect ratio structures of metal oxides. The process starts with the fabrication of a silicon trench template using deep reactive ion etching, and the template is conformal-coated with an AZO film using ALD. The thickness of the coating should be at least half of the maximum distance between the Si trenches to fill the spacing between them. The top part of the AZO coatings can be removed by dry etching using Ar+ ion sputtering. Then, the silicon layers can be etched away selectively by a conventional $\mathrm{SF}_{6}$-based isotropic Si dry etch process. Supporting Information 
Figure S1 shows a schematic of the described fabrication flow. This approach enables us to fabricate high-quality optical metamaterials with high aspect ratio $(20: 1)$ on $2 \times 2 \mathrm{~cm}^{2}$ or even larger areas.

\section{FTIR SPECTROMETRY AND FITTING}

The reflectance spectrum of a $100 \mathrm{~nm}$ thick AZO film deposited on a double-sided polished silicon wafer is measured at $12^{\circ}$ incident angle using a VERTEX 70 FTIR spectrometer from Bruker (Supporting Information, Figure S2). The measurements were performed at five different points on the sample to obtain averaged data. The reflectance from the sample is calculated using the intensity transfer matrix method $^{66}$ and the Drude model for the permittivity of AZO. The calculated reflectance spectrum is then fitted to the measured one using an algorithm based on the LevenbergMarquardt method ${ }^{67}$ to find the parameters of the Drude model (high-frequency dielectric constant, plasma frequency, and damping) for AZO (Supporting Information, Table S4). Our AZO films prepared by ALD deposition exhibit a zero crossing wavelength of around $1.8-2.0 \mu \mathrm{m}$ for highly doped samples. $^{57}$

A similar procedure is carried out for both $\mathrm{AZO} / \mathrm{Si}$ and $\mathrm{AZO} /$ air trench structures where samples are oriented so that the electric field is parallel to the trenches when measuring the reflectance of ordinary waves and perpendicular to the trenches when measuring the reflectance of extraordinary waves (Supporting Information, Figure S3). Considering the Drude-Lorentz model for the ordinary permittivity and the Lorentz model for the extraordinary permittivity, we obtained the effective permittivities (Supporting Information, Table S5 and Figure S4).

\section{THEORETICAL ANALYSIS}

The reflectance maps in Figure 2-4, and Supporting Information Figure S5-S7, are calculated using the transfer matrix method for anisotropic media. ${ }^{68}$ The components of the effective permittivity tensor are restored from the measurement of the reflectance spectra for different polarizations of the incident wave. The profiles of electric field intensity in Figures 2 and 4 are calculated using full-wave numerical simulations with Comsol Multiphysics (COMSOL AB, Stockholm, Sweden). Because of translation symmetry of the structures along the trenches, we simulated the model in $2 \mathrm{D}$ geometry $(x-z$ plane in Figure 1a). The simulation domain consists of a single unit cell with the Floquet boundary conditions. The incident wave is set through the port boundary condition. The infinite thickness of the substrate is simulated by adding a perfectly matched layer.

\section{PRISM COUPLING EXPERIMENT}

Our experimental setup is based on the Otto configuration (see Figure 1a) mounted on an FTIR spectrometer (VERTEX 70, Bruker). We adopted the Otto configuration because the Kretchmann configuration is not feasible due to the thick $\mathrm{Si}$ substrate. A hemispherical $\mathrm{ZnSe}$ prism is placed on the sample with an unavoidable air gap between the prism and the trench structures. The $\mathrm{ZnSe}$ prism is used due to its high refractive index and transparency in the mid-IR region (transparency window is between 0.6 and $17.0 \mu \mathrm{m}$, and its refractive index ranges from 2.60 to 2.35 ). The measurements were conducted in the wavelength range of $\lambda=2.0-16.6 \mu \mathrm{m}\left(5000-602 \mathrm{~cm}^{-1}\right)$ for both TE- and TM-polarized incident light for the three different structures as shown in Supporting Information, Figures S8-S13. A wire grid polarizer is used for controlling the incident polarization. The input light from the thermal light source of the FTIR spectrometer is linearly polarized in either TM polarization, the magnetic field in the $x-y$ plane, or in TE polarization, the electric field in the $x-y$ plane. The beam is focused on the structure through a parabolic mirror and the $\mathrm{ZnSe}$ prism.

\section{ASSOCIATED CONTENT}

\section{S Supporting Information}

The Supporting Information is available free of charge on the ACS Publications website at DOI: 10.1021/acsphotonics.7b00924.

Details of the fabrication procedure of AZO trench structures, optical characterization of AZO films, numerical analysis of surface and bulk waves, and experimental results of the Otto configuration for all samples (PDF)

\section{AUTHOR INFORMATION}

\section{Corresponding Author}

*E-mail: alav@fotonik.dtu.dk.

\section{ORCID}

Osamu Takayama: 0000-0003-3525-3262

Andrei V. Lavrinenko: 0000-0001-8853-2033

\section{Author Contributions}

O.T. and A.V.L. conceived the problem. E.S. developed and conducted the fabrication procedure with the support of R.M. and F.J.; O.T. and E.S. performed characterization of waves supported by the structures. A.B., K.G., P.D., and T.R. performed computational analyses and simulations. M.E.A.P. performed the permittivity retrieval process. All authors participated in interpretation of results and writing the manuscript.

\section{Notes}

The authors declare no competing financial interest.

\section{ACKNOWLEDGMENTS}

Technological and experimental part of this work was supported by Villum Fonden "Blokstipendium", "DarkSILD project No. 11116", and Direktør Ib Henriksens Fond, Denmark. Theoretical analysis was carried out with the support of the Russian Science Foundation (No. 17-19-01731). Modeling was carried out with the support of the Ministry of Education and Science of the Russian Federation (3.1668.2017/4.6). The authors thank Vladimir M. Shalaev, Alexandra Boltasseva, and Thomas Søndergaard for fruitful discussion and valuable comments.

\section{REFERENCES}

(1) Polo, J. A.; Lakhtakia, A. Surface Electromagnetic Waves: A Review. Laser Photon. Rev. 2011, 5, 234-246.

(2) Takayama, O.; Bogdanov, A. A.; Lavrinenko, A. V. Photonic Surface Waves on Metamaterials Interfaces. J. Phys.: Condens. Matter 2017, 29, 463001.

(3) Kildishev, A. V.; Boltasseva, A.; Shalaev, V. M. Planar Photonics with Metasurfaces. Science 2013, 339, 1232009.

(4) Barnes, W. L.; Dereux, A.; Ebbesen, T. W. Surface Plasmon Subwavelength Optics. Nature 2003, 424, 824-830. 
(5) Homola, J.; Yee, S. S.; Gauglitz, G. Surface Plasmon Resonance Sensors: Review. Sens. Actuators, B 1999, 54, 3-15.

(6) Gramotnev, D. K.; Bozhevolnyi, S. I. Nanofocusing of Electromagnetic Radiation. Nat. Photonics 2014, 8, 13-22.

(7) Kawata, S.; Inouye, Y.; Verma, P. Plasmonics for near-Field Nano-Imaging and Superlensing. Nat. Photonics 2009, 3, 388-394.

(8) Vinogradov, A. P.; Dorofeenko, A. V.; Merzlikin, A. M.; Lisyansky, A. a. Surface States in Photonic Crystals. Phys.-Usp. 2010, 53, 243-256.

(9) Descrovi, E.; Sfez, T.; Quaglio, M.; Brunazzo, D.; Dominici, L.; Michelotti, F.; Herzig, H. P.; Martin, O. J. F.; Giorgis, F. Guided Bloch Surface Waves on Ultrathin Polymeric Ridges. Nano Lett. 2010, 10, 2087-2091.

(10) Sinibaldi, A.; Danz, N.; Descrovi, E.; Munzert, P.; Schulz, U.; Sonntag, F.; Dominici, L.; Michelotti, F. Direct Comparison of the Performance of Bloch Surface Wave and Surface Plasmon Polariton Sensors. Sens. Actuators, B 2012, 174, 292-298.

(11) Yu, L.; Barakat, E.; Sfez, T.; Hvozdara, L.; Di Francesco, J.; Peter Herzig, H. Manipulating Bloch Surface Waves in 2D: A Platform Concept-Based Flat Lens. Light: Sci. Appl. 2014, 3, e124.

(12) D'yakonov, M. I. New Type of Electromagnetic Wave Propagating at an Interface. Sov Phys. JETP 1988, 67, 714-716.

(13) Takayama, O.; Crasovan, L.; Artigas, D.; Torner, L. Observation of Dyakonov Surface Waves. Phys. Rev. Lett. 2009, 102, 2-5.

(14) Noginov, M. A. Nano-Optics: Steering Dyakonov-like Waves. Nat. Nanotechnol. 2014, 9, 414-415.

(15) Takayama, O.; Artigas, D.; Torner, L. Lossless Directional Guiding of Light in Dielectric Nanosheets Using Dyakonov Surface Waves. Nat. Nanotechnol. 2014, 9, 419-424.

(16) Pulsifer, D. P.; Faryad, M.; Lakhtakia, A. Observation of the Dyakonov-Tamm Wave. Phys. Rev. Lett. 2013, 111, 1-5.

(17) Kaliteevski, M.; Iorsh, I.; Brand, S.; Abram, R. A.; Chamberlain, J. M.; Kavokin, A. V.; Shelykh, I. A. Tamm Plasmon-Polaritons: Possible Electromagnetic States at the Interface of a Metal and a Dielectric Bragg Mirror. Phys. Rev. B: Condens. Matter Mater. Phys. 2007, 76, 165415.

(18) Sasin, M. E.; Seisyan, R. P.; Kaliteevski, M. A.; Brand, S.; Abram, R. A.; Chamberlain, J. M.; Iorsh, I. V.; Shelykh, I. A.; Egorov, A. Y.; Vasil'ev, A. P.; Mikhrin, V. S. Tamm Plasmon-Polaritons: First Experimental Observation. Superlattices Microstruct. 2010, 47, 44-49.

(19) Cai, W.; Shalaev, V. Optical Metamaterials: Fundamentals and Applications; Springer-Verlag: New York, NY, 2010.

(20) Yu, N.; Capasso, F. Flat Optics with Designer Metasurfaces. Nat. Mater. 2014, 13, 139-150.

(21) Jahani, S.; Jacob, Z. All-Dielectric Metamaterials. Nat. Nanotechnol. 2016, 11, 23-36.

(22) Jacob, Z.; Narimanov, E. E. Optical Hyperspace for Plasmons: Dyakonov States in Metamaterials. Conf. Proc. - Lasers Electro-Optics Soc. Annu. Meet. 2009, 563-564.

(23) Takayama, O.; Artigas, D.; Torner, L. Practical Dyakonons. Opt. Lett. 2012, 37, 4311-4313.

(24) Poddubny, A.; Iorsh, I.; Belov, P.; Kivshar, Y. Hyperbolic Metamaterials. Nat. Photonics 2013, 7, 948-957.

(25) Smith, D. R.; Schurig, D. Electromagnetic Wave Propagation in Media with Indefinite Permittivity and Permeability Tensors. Phys. Rev. Lett. 2003, 90, 77405.

(26) Sun, J.; Litchinitser, N. M.; Zhou, J. Indefinite by Nature: From Ultraviolet to Terahertz. ACS Photonics 2014, 1, 293-303.

(27) Guo, Y.; Newman, W.; Cortes, C. L.; Jacob, Z. Applications of Hyperbolic Metamaterial Substrates. Adv. OptoElectron. 2012, 2012, $1-9$.

(28) Ferrari, L.; Wu, C.; Lepage, D.; Zhang, X.; Liu, Z. Hyperbolic Metamaterials and Their Applications. Prog. Quantum Electron. 2015, 40, 1-40.

(29) Lu, D.; Liu, Z. Hyperlenses and Metalenses for Far-Field SuperResolution Imaging. Nat. Commun. 2012, 3, 1205.

(30) Kabashin, V.; Evans, P.; Pastkovsky, S.; Hendren, W.; Wurtz, G. a; Atkinson, R.; Pollard, R.; Podolskiy, V. a; Zayats, a V. Plasmonic Nanorod Metamaterials for Biosensing. Nat. Mater. 2009, 8, 867-871.
(31) Sreekanth, K. V.; Alapan, Y.; ElKabbash, M.; Ilker, E.; Hinczewski, M.; Gurkan, U. A.; De Luca, A.; Strangi, G. Extreme Sensitivity Biosensing Platform Based on Hyperbolic Metamaterials. Nat. Mater. 2016, 15, 621-627.

(32) Kruk, S. S.; Wong, Z. J.; Pshenay-Severin, E.; O’Brien, K.; Neshev, D. N.; Kivshar, Y. S.; Zhang, X. Magnetic Hyperbolic Optical Metamaterials. Nat. Commun. 2016, 7, 11329.

(33) Kapitanova, P. V.; Ginzburg, P.; Rodríguez-Fortuño, F. J.; Filonov, D. S.; Voroshilov, P. M.; Belov, P. a; Poddubny, A. N.; Kivshar, Y. S.; Wurtz, G. a; Zayats, A. V. Photonic Spin Hall Effect in Hyperbolic Metamaterials for Polarization-Controlled Routing of Subwavelength Modes. Nat. Commun. 2014, 5, 3226.

(34) High, A. A.; Devlin, R. C.; Dibos, A.; Polking, M.; Wild, D. S.; Perczel, J.; De Leon, N. P.; Lukin, M. D.; Park, H. Visible-Frequency Hyperbolic Metasurface. Nature 2015, 522, 192-196.

(35) Avrutsky, I.; Salakhutdinov, I.; Elser, J.; Podolskiy, V. Highly Confined Optical Modes in Nanoscale Metal-Dielectric Multilayers. Phys. Rev. B: Condens. Matter Mater. Phys. 2007, 75, 2-5.

(36) Krishnamoorthy, H. N. S.; Jacob, Z.; Narimanov, E.; Kretzschmar, I.; Menon, V. M. Topological Transitions in Metamaterials. Science 2012, 336, 205-209.

(37) Naik, G. V.; Saha, B.; Liu, J.; Saber, S. M.; Stach, E. a; Irudayaraj, J. M. K.; Sands, T. D.; Shalaev, V. M.; Boltasseva, A. Epitaxial Superlattices with Titanium Nitride as a Plasmonic Component for Optical Hyperbolic Metamaterials. Proc. Natl. Acad. Sci. U. S. A. 2014, $111,7546-7551$.

(38) Maas, R.; Parsons, J.; Engheta, N.; Polman, A. Experimental Realization of an Epsilon-near-Zero Metamaterial at Visible Wavelengths. Nat. Photonics 2013, 7, 907-912.

(39) Smalley, J. S. T.; Vallini, F.; Montoya, S. A.; Ferrari, L.; Shahin, S.; Riley, C. T.; Kanté, B.; Fullerton, E. E.; Liu, Z.; Fainman, Y. Luminescent Hyperbolic Metasurfaces. Nat. Commun. 2017, 8, 13793.

(40) Noginov, M. A.; Barnakov, Y. A.; Zhu, G.; Tumkur, T.; Li, H.; Narimanov, E. E. Bulk Photonic Metamaterial with Hyperbolic Dispersion. Appl. Phys. Lett. 2009, 94, 1-4.

(41) Riley, C. T.; Smalley, J. S. T.; Post, K. W.; Basov, D. N.; Fainman, Y.; Wang, D.; Liu, Z.; Sirbuly, D. J. High-Quality, Ultraconformal Aluminum-Doped Zinc Oxide Nanoplasmonic and Hyperbolic Metamaterials. Small 2016, 12, 892-901.

(42) Gomez-Diaz, J. S.; Tymchenko, M.; Alù, A. Hyperbolic Plasmons and Topological Transitions over Uniaxial Metasurfaces. Phys. Rev. Lett. 2015, 114, 233901.

(43) Zhong, Y.; Wasserman, D.; Zhong, Y.; Malagari, S. D.; Hamilton, T.; Wasserman, D. Review of Mid-Infrared Plasmonic Materials Review of Mid-Infrared Plasmonic Materials. J. Nanophotonics 2015, 9, 93791.

(44) Haas, J.; Mizaikoff, B. Advances in Mid-Infrared Spectroscopy for Chemical Analysis. Annu. Rev. Anal. Chem. 2016, 9, 45-68.

(45) Naik, G. V.; Shalaev, V. M.; Boltasseva, A. Alternative Plasmonic Materials: Beyond Gold and Silver. Adv. Mater. (Weinheim, Ger.) 2013, $25,3264-3294$

(46) Caldwell, J. D.; Vurgaftman, I.; Tischler, J. G.; Glembocki, O. J.; Owrutsky, J. C.; Reinecke, T. L. Atomic-Scale Photonic Hybrids for Mid-Infrared and Terahertz Nanophotonics. Nat. Nanotechnol. 2016, $11,9-15$.

(47) Basov, D. N.; Fogler, M. M.; Garcia de Abajo, F. J. Science (Washington, DC, U. S.) 2016, 354, aag1992-aag1992.

(48) Low, T.; Chaves, A.; Caldwell, J. D.; Kumar, A.; Fang, N. X.; Avouris, P.; Heinz, T. F.; Guinea, F.; Martin-Moreno, L.; Koppens, F. Polaritons in Layered 2D Materials. Nat. Mater. 2017, 16, 182-194.

(49) Caldwell, J. D.; Lindsay, L.; Giannini, V.; Vurgaftman, I.; Reinecke, T. L.; Maier, S. A.; Glembocki, O. J. Low-Loss, Infrared and Terahertz Nanophotonics Using Surface Phonon Polaritons. Nanophotonics 2015, 4, 44-68.

(50) Caldwell, J. D.; Glembocki, O. J.; Francescato, Y.; Sharac, N.; Giannini, V.; Bezares, F. J.; Long, J. P.; Owrutsky, J. C.; Vurgaftman, I.; Tischler, J. G. Low-Loss, Extreme Subdiffraction Photon Confinement via Silicon Carbide Localized Surface Phonon Polariton Resonators. Nano Lett. 2013, 13, 3690-3697. 
(51) Gubbin, C. R.; Martini, F.; Politi, A.; Maier, S. A.; De Liberato, S. Strong and Coherent Coupling between Localized and Propagating Phonon Polaritons. Phys. Rev. Lett. 2016, 116, 1-6.

(52) Talebi, N.; Ozsoy-Keskinbora, C.; Benia, H. M.; Kern, K.; Koch, C. T.; Van Aken, P. A. Wedge Dyakonov Waves and Dyakonov Plasmons in Topological Insulator Bi2Se3 Probed by Electron Beams. ACS Nano 2016, 10, 6988-6994.

(53) Li, P.; Dolado, I.; Alfaro-Mozaz, F. J.; Nikitin, A. Y.; Casanova, F.; Hueso, L. E.; Vélez, S.; Hillenbrand, R. Optical Nanoimaging of Hyperbolic Surface Polaritons at the Edges of van Der Waals Materials. Nano Lett. 2017, 17, 228-235.

(54) Michelotti, F.; Dominici, L.; Descrovi, E.; Danz, N.; Menchini, F. Thickness Dependence of Surface Plasmon Polariton Dispersion in Transparent Conducting Oxide Films at 1.55 Microm. Opt. Lett. 2009, 34, 839-841.

(55) Guske, J. T.; Brown, J.; Welsh, A.; Franzen, S. Infrared Surface Plasmon Resonance of AZO-Ag-AZO Sandwich Thin Films. Opt. Express 2012, 20, 23215.

(56) George, S. Atomic Layer Deposition: An Overview. Chem. Rev. (Washington, DC, U. S.) 2010, 110, 111-131.

(57) Shkondin, E.; Takayama, O.; Panah, M. E. A.; Liu, P.; Larsen, P. V.; Mar, M. D.; Jensen, F.; Lavrinenko, A. V. Large-Scale High Aspect Ratio Al-Doped $\mathrm{ZnO}$ Nanopillars Arrays as Anisotropic Metamaterials. Opt. Mater. Express 2017, 7, 1606-1627.

(58) Alam, M. Z.; De Leon, I.; Boyd, R. W.; Kauranen, M.; Zayats, A. V.; Abb, M.; Albella, P.; Aizpurua, J.; Muskens, O. L.; Neira, A. D. Large Optical Nonlinearity of Indium Tin Oxide in Its Epsilon-nearZero Region. Science 2016, 352, 795-797.

(59) Caspani, L.; Kaipurath, R. P. M.; Clerici, M.; Ferrera, M.; Roger, T.; Kim, J.; Kinsey, N.; Pietrzyk, M.; Di Falco, A.; Shalaev, V. M. Enhanced Nonlinear Refractive Index in Epsilon -Near-Zero Materials. Phys. Rev. Lett. 2016, 116, 1-5.

(60) Belov, P. A.; Simovski, C. R.; Ikonen, P. Canalization of Subwavelength Images by Electromagnetic Crystals. Phys. Rev. B: Condens. Matter Mater. Phys. 2005, 71, 193105.

(61) Feigenbaum, E.; Diest, K.; Atwater, H. A. Unity-Order Index Change in Transparent Conducting Oxides at Visible Frequencies. Nano Lett. 2010, 10, 2111-2116.

(62) Dominici, L.; Auf Der Maur, M.; Michelotti, F. Strong FreeCarrier Electro-Optic Response of Sputtered ZnO Films. J. Appl. Phys. (Melville, NY, U. S.) 2012, 112, 53514.

(63) Lee, H. W.; Papadakis, G.; Burgos, S. P.; Chander, K.; Kriesch, A.; Pala, R.; Peschel, U.; Atwater, H. A. Nanoscale Conducting Oxide PlasMOStor. Nano Lett. 2014, 14, 6463-6468.

(64) Rodrigo, D.; Limaj, O.; Janner, D.; Etezadi, D.; de Abajo, F. J. G.; Pruneri, V.; Altug, H. Mid-Infrared Plasmonic Biosensing with Graphene. Science 2015, 349, 165-168.

(65) Michelotti, F.; Sciacca, B.; Dominici, L.; Quaglio, M.; Descrovi, E.; Giorgis, F.; Geobaldo, F. Fast Optical Vapour Sensing by Bloch Surface Waves on Porous Siliconmembranes. Phys. Chem. Chem. Phys. 2010, 12, 502-506.

(66) Gabriel, C. J.; Nedoluha, A. Transmittance and Reflectance of Systems of Thin and Thick Layers. Opt. Acta 1971, 18, 415-423.

(67) Press, W.; Flannery, B.; Teukolsky, S.; Vetterling, W. Numerical Recipes; Cambridge University Press: Cambridge, NY, 1986.

(68) Yeh, P. Optics of Anisotropic Layered Media: A New $4 \times 4$ Matrix Algebra. Surf. Sci. 1980, 96, 41-53. 